intra-dimer contacts and the formation of oligomers that consist of greater numbers of units (Protein Data Bank accession number, $1 \mathrm{~N} 3 \mathrm{M})$.

Dimitrios Fotiadis ${ }^{\star}$, Yan Liang $\dagger$,

Slawomir Filipek‡, David A. Saperstein $\dagger$, Andreas Engel ${ }^{\star}$, Krzysztof Palczewski $\uparrow \$ \|$

${ }^{\star}$ M. E. Müller Institute for Microscopy,

Biozentrum, University of Basel, Basel 4056,

Switzerland

Departments of $\dagger$ Ophthalmology, \$Pharmacology and |Chemistry, University of Washington, Seattle, Washington 98195, USA

\section{Coulomb fission}

\section{Rayleigh jets from levitated microdroplets}

Et lectrified droplets are generated in thunderstorm clouds, as well as in technological applications such as inkjet printing and electrospray ionization, but they become unstable when charged beyond the Rayleigh limit ${ }^{1}$. Here we record the dynamics of the disintegration process by examining levitated droplets under high-speed microscopy. These images may help to explain one of the oldest unsolved problems in experimental and theoretical physics.

Lord Rayleigh showed that the spherical shape of a drop of radius $a_{0}$, surface tension $\sigma$ and charge $Q$, remains stable as long as the fissility $X=Q^{2} /\left(64 \pi^{2} \epsilon_{0} \sigma a_{0}{ }^{3}\right)$ does not exceed unity ${ }^{1}$. As $X$ approaches unity, the quadrupole deformation is the first to become unstable; when $X$ increases beyond unity, however, an instability occurs that is associated with the formation of fine jets

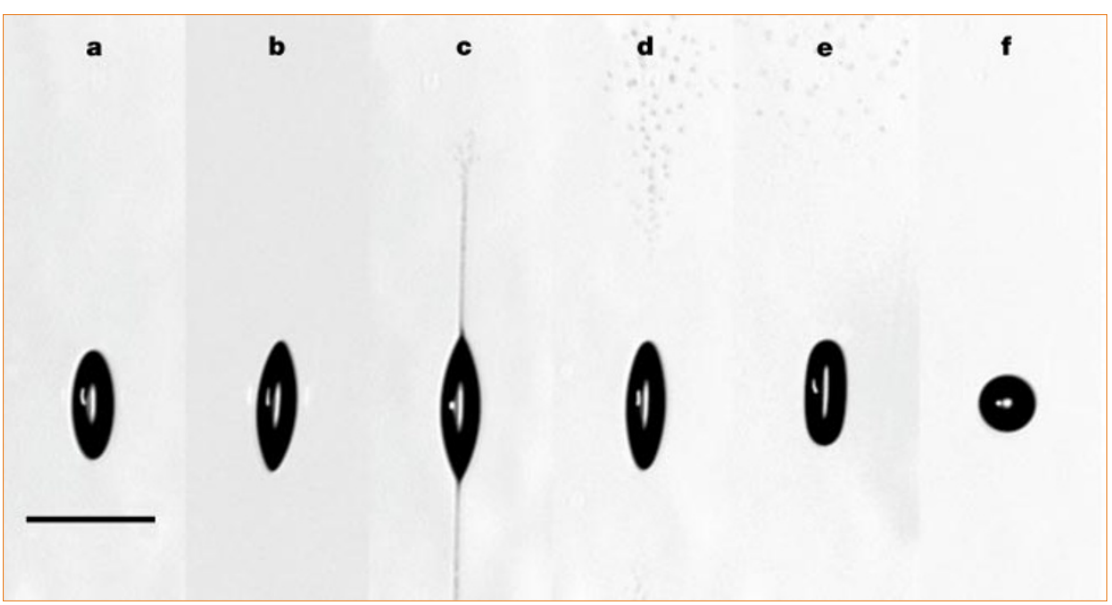

Figure 1 High-speed imaging of the disintegration of a levitated droplet charged to the Rayleigh limit. The droplet (radius, $24 \mu \mathrm{m}$ ) is imaged on a vertical charge-coupled-device array to determine and control its vertical position in the levitator, and on a photomultiplier that detects instability-onset, quadrupole-shaped oscillations of the droplet. These oscillations trigger a signal to a flashlamp that fires after a predefined delay, $\Delta t$. The image is observed through a microscope with a long working distance (Mitutoyo). a-f, Microscopic images taken at $\Delta t$ values (in $\mu$ s) of: a, 140; b, 150; c, 155; d, 160; e, 180, and f, 210. The droplet changes from a sphere to an ellipsoid (a), tips appear at the poles (b) and a fine jet of liquid is ejected from each tip (c); the jets disintegrate (d) and the elliptical droplet reassumes a spherical shape $(\mathbf{e}, \mathbf{f})$. Further experimental details are available from the authors. Scale bar, $100 \mu \mathrm{m}$. shaped oscillation of the droplet increases drastically ${ }^{9}$, triggering a signal to a fast flashlamp that fires after a predefined delay, $\Delta t$, and is recorded by a digital microscope as a still image of the droplet. This process is repeated with subsequent droplets, using increasing delay times.

These microscopic images of the disintegration process of our levitated microdroplets are reproduced in Fig. 1a-f. During the first $150 \mu \mathrm{s}$, the droplet stretches from a sphere into an ellipsoid, as predicted by Rayleigh (Fig. 1a). It then develops two sharp tips on the poles of the ellipsoid (Fig. 1b). Almost instantaneously after tip formation, a fine jet of liquid is ejected from both tips in opposite directions (Fig. 1c). This jet later disintegrates into fine droplets (Fig. 1d) that are repelled from the mother droplet by Coulomb repulsion. The tip edges disappear after the ejection of the jet (Fig. 1e), and the barrelshaped parent droplet then contracts until it regains spherical symmetry after about $210 \mu \mathrm{s}$ (Fig. 1f).

During the jet's disintegration, roughly 100 small daughter droplets are formed, which carry $33 \%$ of the total charge and constitute about $0.3 \%$ of the mass of the mother droplet. The diameter of the jet is determined from higher-resolution images to be $1.5 \mu \mathrm{m}$ : the droplets generated during disintegration are of roughly the same size and are themselves close to a fissility of $X_{\text {frag }}=1$ and so presumably undergo a Rayleigh instability soon afterwards.

As this behaviour is independent of the levitator voltage, we argue that it must correspond closely to that of free, highly charged droplets. But, in contrast to Lord Rayleigh's prediction, we observed the jets at a fissility of unity, indicating that renewed investigation will be necessary to explain the complex hydrodynamics of this century-old problem.

Denis Duft, Tobias Achtzehn, Rene Müller, Bernd A. Huber ${ }^{\star}$, Thomas Leisner Institut für Physik, Technische Universität Ilmenau, Postfach 100565, 98684 Ilmenau, Germany e-mail: thomas.leisner@TU-Ilmenau.de ${ }^{*}$ Permanent address: Centre Interdisciplinaire de Recherche Ions Lasers, rue Claude Bloch, 14070 Caen Cedex 5, France

1. Rayleigh, Lord Phil. Mag. 14, 184-186 (1882)

2. Taylor, G. I. Proc. R. Soc. Lond. A 280, 383-397 (1964).

3. Smith, J. N., Flagan, R. C. \& Beauchamp, J. L. J. Phys. Chem. A 106, 9957-9967 (2002).

4. Davis, E. J. \& Schweiger, G. The Airborne Microparticle (Springer, Heidelberg, 2002).

5. Tsampoulos, J. A., Akylas, T. R. \& Brown, R. A. Proc. R. Soc. Lond. A 401, 67-88 (1985).

6. Pashkevich, V. V., Krappe, H. J. \& Wehner, J. Z. Phys. D 40, 338-340 (1997).

7. Krämer, B. et al. J. Chem. Phys. 111, 6521-6527 (1999).

8. Schwell, M. et al. J. Phys. Chem. A 104, 6726-6732 (2000)

9. Duft, D., Lebius, H., Huber, B. A., Guet, C. \& Leisner, T. Phys. Rev. Lett. 89, 0845031-0845034 (2002).

Competing financial interests: declared none. 